\title{
Meta
}

Journal des traducteurs

Translators' Journal

\section{La problématique de la traduction théâtrale et de l'adaptation au Québec}

\section{Michèle Laliberté}

Volume 40, numéro 4, décembre 1995

URI : https://id.erudit.org/iderudit/003377ar

DOI : https://doi.org/10.7202/003377ar

Aller au sommaire du numéro

Éditeur(s)

Les Presses de l'Université de Montréal

ISSN

0026-0452 (imprimé)

1492-1421 (numérique)

Découvrir la revue

Citer cet article

Laliberté, M. (1995). La problématique de la traduction théâtrale et de l'adaptation au Québec. Meta, 40(4), 519-528. https://doi.org/10.7202/003377ar

\section{Résumé de l'article}

Cet article fait une mise au point sur l'emploi du québécois et du joual dans les traductions théâtrales au Québec. L'auteur, à partir de problèmes de traduction liés à l'analyse de la pièce de Fasshintler « Der Müll, die Stadt und der Tod », étudie le phénomène de l'adaptation théâtrale et démontre la nécessité d'intégrer les traductions théâtrales. 


\title{
LA PROBLÉMATIQUE DE LA TRADUCTION THEATTRALE ET DE L'ADAPTATION AU QUEBBEC
}

MiCHËLE LALIBERTE

Trinisy College. Burlington, Etars-Unis

\begin{abstract}
Résumé
Cet article fait une mise au point sur l'emploi du québécois et du joual dans les traductions theâtrales au Québec' L'auseur. d partir de problèmes de traduction liés a l'analyse de la pièce de Fasshinder "Der Müll, die Stadt und der Tod"e etudic le phénomène de ladaptation théatrale é démontre la nécessifé d' intégrer les traductions théâtrales.
\end{abstract}

Abstract

This article examines the use of Quebeccois and joual in theatre translation in Quebec: Referring w her difficulties translating Fasshinder's play Der Müll, die Stadt und der Tod. the author looks at theatre adaptation and show's the need to integrate play translations inso the social conted of the target andience.

En traduisant la dernière pièce de Rainer Werner Fassbinder. Der Müll, die Stadt und der Tod, mon intention première était de demeurer fidèle au texte de départ ainsi qu'à la pensée de l'auteur. J'étais loin de $m$ 'imaginer que ce travail pouvait à lui seul me faire changer tant de fois d'idées sur la façon de traduire un texte théâtral pour un public inontréalais. De prime abord, je ne voulais pas adapter le texte de Fassbinder. J'ai toujours eu un préjugé défavorable en ce qui conceme les adaptations qui transposent l'action en dehors de leur contexte original. À mon avis, de pareilles traductions ne pouvaient que Irahir l'cuvre originale. Cependant, il était clair que je ne voulais pas faire parler les prostituées de la pièce en argot parisien. Je traduisais cette ceuvre pour qu'on puisse la jouer à Montréal. Il me fallait donc traduire pour un public montréalais, en essayant de respecter le plus possible les niveaux de langue de l'original. L'essentiel était de traduire cette pièce à voix haute, afin de respecter le rythme et de rendre l'expression de l'affect plausible. Il était donc primordial de faire en sorte que le spectateur ne se rende pas compte que la pièce avait été traduite. Je tenais à ce qu'il ait l'impression qu'elle avait été Écrite directement en québécois. Tout résidait donc dans la question du style :

The first principle of play Iranslation is style. By style I mean the quality that conceals a translation's provenance. In other words, style is that which causes a play to sound as if it had originally been written in the target language. |... Translation is the re'-creation of the original language's meaning in the syntax and, in the case of a modern work, in the socially accepted style of the target language. (Wellwarth 1981:142)

Évidemment. tous ne sont pas d'accord avec ce point de vue. Berman (1985: 79), notamment, écrit que :

L'exotisation peut rejoindre la vulgarisation en rendant un vernaculaire étranger par un vernaculaire local |... |. Malheureusement, le vernaculaire ne peut être traduit dans un autre vernaculaire. Seules les koinès, les langues cultivées, peuvent s'entretraduire. Une telle exotisation, qui rend l'étranger du dehors par celui du dedans, $n$ 'aboutit qu'à ridiculiser l'original. 
À mon avis, cette affirmation dérive directement de cette attitude méprisante envers les langues vernaculaires qu'entretient jalousement l'élite intellectuelle française. On ne retrouve pas de pareilles affirmations dans le monde anglophone. De plus, en ce qui conceme le théâtre. où «le public, la culture-cible, $|\ldots|$ vérifient immédiatement si le texte passe ou non!» (Pavis 1987: 420), on est en droit de voir les choses d'un autre ail. Cependant, lorsqu' on choisit de traduire en ce qui est considéré comme la langue ades gens qu'une faible ou mauvaise scolarisation protège mal des quotidiens assauts perpétrés contre leur culture" (Lalonde 1979: 29), il nous faut, même en 1995, justifier cette démarche. Non, je n'ai pas choisi ce parler populaire pour essayer ade faire honte à nos élites bourgeoises trop bien pensantes ou bien parlantes» ou pour "ré-identifier fla culture québécoise ||$\ldots \mid$ à la majorité prolétarisée de la nation» (/hid. : 29). Il se trouve que kes personnages de cette pièce font partie de cette majorité prolétarisée. J'ai quand même hésité. Après une année entière de tergiversations, après avoir dû maintes fois réviser ma perspective traductologique, $\mathrm{j}$ 'ai décidé d'adapter. Les pages qui suivent tenteront de démontrer pourquoi, a mon avis, il le fallait. Dans un premier temps. cependant. j'aimerais tenter de faire le point sur l'emploi du québécois et du joual en traduction théấtrale. Puis, à partir de mes propres problèmes de traduction. j'essaierai d'étudier le phénomène de l'adaptation, auquel j'étais tout d'abord réfractaire au plus haut point.

Faut-il traduire le discours des personnages de pièces de théâtre en québécois, ou même en joual selon le cas? Il apparaît que les opinions sont partagées. La journaliste Lysianne Gagnon écrivait dans La Pressé, à propos de l'Ééll du printemps. de Frank Wedekind, dont la traduction avait été faite par Jean-Luc Denis :

Comme la pièce reste située dans le contexte de l'Allemagne au toumant du siècle. elle devrait normalement être traduite dans la langue la plus neutre possible. en français standard. (Le français standard, rappelons-le, n'est pas nécessairement celui de Paris : c'est la langue de Bernard Derome ou de Richard Garneau, qui peut être comprise parout dans le monde francophone.) Mais les acteurs parlent comme si l'action se passait sur le Plateau MontRoyal. Le résultat est évidemment incongru. (Gagnon 1989: B.3)

René Gingras (1990): 32), dans un numéro spécial de la revue Je'u sur la traduction théâtrale, résume sa pensée en ces termes:

Je trouve dommage qu ion se retrouve encore une fois à faire vraiment une différence entre la France et le Québec. Il me semble qu'on a une responsabilité. non seulement envers le français qui se parle ici, mais aussi envers la langue française. Je considere qu'on parle français et qu'on a quelque chose à apponer à la langue française au même litre que la langue qui est parlée en France. En refusant toute concession parce qu'on est Québécois et qu'il est évident qu'on écrit en québécois, je trouve qu'on perpétue une séparation qu'il faudrait peut-être travailler à élaguer. Quand on a à choisir, par exemple, entre deux mots dont on sait que l'un sera compris ailleurs et l'autre pas, j’avoue avoir un préjugé favorable pour la façon de dire qui sera comprise ici et ailleurs.

Pour Annic Brisset, le discours étranger a été, au Québec, utilisé dans un but d' «émancipation nationale». Comme elle le démontre remarquablement dans son livre intitulé Sociocritique de la traduction. Théâtre et altérité au Québec (1968-1988). I'usage du québécois en traduction théâtrale est né de ce besoin qu avaient les Québécois de se différencier du Français (l’impérialiste culturel) et de l'Anglais (l'impérialiste économique). De la naissance du Parti québécois à l'échec de l'accord du lac Meech. c'est la préoccupation identitaire qui est au centre du discours social. Le théâtre doit donc éveiller chez. le spectateur "la conscience d'être Québécois". C"est ainsi qu'on en est arrivé à adapter (c'est-à-dire à transposer complètement) la célèbre pièce de Brecht $D i e$ Kleinhürgerhochzeit, en lui donnant pour titre La nôsse chez les mopricituires de hungalsu. 
(Brecht 1976)'. Cette adaptation est un exemple de cette tendance adoptée par le Québec des années soixante-dix qui consistait à transformer l'altérité pour servir la cause nationaliste. On parle ici d'adaptation. Il faudrait tout d'abord s'interroger sur le sens de ce mot, qui peut avoir une connotation péjorative lorsqu'il s'agit de traduction théâtrale. L.e premier sens du mot adaptation, selon Jean-Michel Déprats, désigne le passage d'un genre à un autre. Pour le traducteur, cependant, ce qui distingue l'adaptation de la traduction (et ce qui correspond à la deuxième définition du terme), c'est que "l'adaptation comporte des coupures, des ajouts ou des restructurations" (Déprats 1990: 38). L'adaptation thêâtrale peut donc être considérée comme un genre en soi. Annie Brisset nous parle de traduction (elle n'établit pas clairement la différence entre adaptation et traduction et elle traite souvent des deux à la fois, sans faire de distinction) et nous dit que la traduction théâtrale au Québec a servi à «effacer l'Autre», et que c'est la langue québécoise qui a été utilisée pour établir cette séparation.

Le français ne suffit plus, car l'enjeu est désormais topologique : la langue doit être coextensive au territoire. L'invention du Soi passe par l'invention d'une langue propre. entreprise à laquelle participe la traduction. (Brisset $1990 \mathrm{c}: 57-58$ )

L'invention d'une langue propre? L'auteure sous-entend ici que le travail sur la langue, qui est fait lors de la traduction d'une pièce de théâtre, consiste à "parasiter l'orthographe du français pour démontrer l'existence et la spécificité de la "langue québécoise"» (Brisset 1990b : 46). En plus de parasiter le français "pour simuler la différence", Annie Brisset affirme que la traduction théâtrale au Québec a reproduit un sociolecte érigé en l.angue nationale (sociolecte qui n'est pourtant parlé que par les couches prolétaires de la société québécoise, affirme-t-elle), et que le fait de traduire en québécois prouve que le Québec est à la recherche d'un code distinctif "qui débouche inévitablement sur la mise en place d'une langue autarcique». L'auteure soutient que, maintenant que le Québec s'est affirmé sur le plan politique, il $n$ 'est nul besoin de recourir à l'adaptation et de situer Roméo et Julietto' sur le bord du Saint-Laurent. De plus, la dramaturgie québécoise s'étant libérée de son devoir de conscientisation nationale. le traducteur n'est plus dans l'obligation d'utiliser une langue "catastrophée". Robert Lévesque (1993: A8) se range du côté d'Annie Brisset lorsqu'il fait le procès du Macherh de Robert Lepage. Il parle d'une I raduction "archi-complaisante" où le québécois "passé d’âge" correspond à cette "poussée de l'affirmation nationale où le simple fait de dire "toé" ou "che nous" était vu comme une conquête des masses joualisantes et nationales». De plus, toujours selon Lévesque (ihid.), ce qu'il y avait de plus incongru dans la traduction de Garneau était le fail que tous les personnages parlaient «de la même manière pas dégrossie. |... le roi Duncan et le soldat de garde, lady Macbeth et les sorcières. comme si tout ce monde-là avait gardé les cochons ensemble». On peut opposer à $M$. Lévesque les arguments suivants : premièrement, il est important de noter que la langue anglaise du XVII ${ }^{e}$ siècle, comme le mentionne Léandre Bergeron (1981:29-30), est une langue vernaculaire parlée par toutes les classes sociales. Les (xuvres de Shakespeare le démontrent d'ailleurs très clairement. L'anglais est la langue de tout le monde. Donc, il n'y avait pas de réelle dichotomie entre le parler du roi et celui de ses serviteurs. Deuxièmement, la dichotomie existant aujourd hui entre le «bon français» et ses dialectes (ainsi que celle existant entre la langue française el québécoise) $n$ 'existe que depuis l'intervention savante de François $I^{e r}$.

En 15.39. François $I^{\mathrm{er}}$ proclame l'ordonnance de Villers-Cotterêts qui fait du français de François la langue officielle du royaume de France... (ibid. : 23)

En 1634. Richelieu élatise la langue en fondant le corps policier pour la régir. II fonde l'Académie française | ... Pendant qu'en France la langue vernaculaire du roi devient langue 
d'État et subit codification et épuration. nos ancêtres perpétuent en terre d'Amérique l'évolution naturelle des vernaculaires de France... (ibid. : 25 et 3.3)

À la lumière de cette citation, ne peut-on pas légitimer la démarche de Garneau ?

Nous pensons que le théâtre n'est pas un genre littéraire mais une pratique scénique, comme l'affirme Anne Ubersfeld (1981: 16). "Au théâtre, les énoncés du dialogue deviennent discours à partir du moment où leur sont données des conditions d'énonciation, l'énonciation étant "la mise en fonctionnement de la langue par un acte individuel d'utilisation" "’2. Ainsi, lorsqu 'on traduit du théatre, il faut traduire pour e'ntendre'. ( "Lire, c'est regarder, cest voir $\mid . .$. | et ce que l'on voit peut être tout autre chose que ce que l'on entend.") (Jourdheuil 1990: 34.) La traduction théâtrale s'avère donc très spécifique : il faut que le langage des personnages soit crédible pour un public spécitique lors de l'énonciation, parce qu'au théâtre, cette énonciation est pourvue d'une connotation affective. "L'objet du contrat est l'échange du plaisir/désir: les conditions de l'échange dépendent de la situation de la communication (par exemple, pour un spectateur, la reconnaissance ou le déni de son imposture...» (Helbo 1983 : 43). Le traducteur québécois utilise un français normatif pour la plupart des textes qu'il a à traduire. Pour le texte thêatral, cependant, où l'expression de l'affect s'avère prépondérante, il est normal que le traducteur ait recours à tous les registres de la langue québécoise, y compris le niveau familier. Il suffit de respecter les niveaux de langue du texte de départ. Si le personnage n'utilise pas les registres de la langue parlée dans le texte de dépan, le traducteur n'aura pas à utiliser la langue intragroupe, pour employer l'expression de Nida (1975: 148).

Pour qui traduit-on le théâtre au Québec ? M. René Gingras décrie l'attitude autonomiste que nous avons envers le français québécois et il insiste sur le fait que nos traductions théâtrales devraient être comprises par les Français ! Est-ce que les Américains traduisent le théâtre en fonction de ce que pourront en comprendre les Anglais? Est-ce que les Mexicains tiennent compte des Espagnols? En Suisse alémanique, on utilise le suisse-allemand pour traduire le théâtre. Il est cependant normal, selon Jean-Luc Denis, que nous ayons quelque difficulté à accepter que le théâtre étranger soit traduit en joual, même si, dans la langue de départ. on utilise un dialecte :

L'habitude détermine ce qui est incongru et ce qui ne l'est pas dans l'accuejl que l’on faut à lal langue utilisée pour traduire un personnage étranger : or. en raison de l'omniprésence du doublage français au cinéma è à la télévision. nous n avons jamais entendu un soldat nazi ou un paysan polonais parler une langue autre que le français dominamt la postsynchronisation parisienne. Toute incursion dans le français régional. si minime soit-elle. est perçue comme un ajout malséant de couleur locale - notre oreille n'y est pas accoutuméce, (Denis 1990): 15)

Il y a bel et bien clivage entre la langue parlée et la langue écrite au Québec : il y a. d'une part. la langue-étalon imposée par l'Ačadémie française et. d'autre part. l'emploi qu'en fait le peuple.

La langue soutenue est celle que l'on apprend à l'école. celle qu'on écrit. mais pas nécessairement celle que l'on parle. [ . . I Signalons, d'ailleurs, yue cette situation n'est pas unique a la francophonie. Flle se retrouve dans presque rous les pays du monde oi une societe fortement hiérarchiséc a permis aux lettrés de vivre en marge du peuple et d'ślaborer une langue a part estiméc supéricure à celle du commun. On la retrouve entre autres en laalice. en (irece. en Chine et dans les pays arabes. Mais, il en est autrement dans les pays scandinaves el les pays anglophones où une longue tradition démocratique a su produire une langue moins clivéc. $|. .$.$| Au fond, la question du niveau de langue se pose surnout au traducteur de theiatre$ qui, lui, dans sa pratique courante, est amené à ecrire dans une langue destiné à être parlée. 
Son média $\mid$ sic $\mid$ est précisément cette langue occultée, non codifiée, qu'il doit apprendre à manier avec le maximum de précision'. (Bélisle $1990: 20-21$ )

Nous sommes tout à fait d'accord avec Annie Brisset lorsqu'elle affirme que le Québec a eu recours, par le passé, à l'adaptation, dans le but d' "effacer l'Autre». La traduction théâtrale était alors investie de préoccupations identitaires. Cependant, là où le bât blesse, c'est lorsqu'elle affirme que certains traducteurs parasitent le français et que notre sociolecte n'est parlé que par les couches prolétaires de la société québécoise.

Toutefois, ce qu'on appelle la langue québécoise diffère assez peu du "français de France», si ce n'est par la phonétique. Cetle différence est d'ailleurs toute relative. Elle n'est vraiment perceptible que dans le parler des classes prolétaires... (Brisset 1990b : 44)

Nous croyons, tout comme Jean-Luc Denis, que la langue française parlée au Québec ne se limite pas à la dyade français normatif-joual. Au contraire, cette langue parlée comprend différents registres sociolinguistiques qui ne sont pas uniquement utilisés par les classes prolétaires. De plus, ces différents niveaux de langue diffèrent, pour la plupart, de la langue parlée en France, tant sur le plan du vocabulaire que de la syntaxe. Tout comme Lefebvre et Ostiguy, nous croyons que le fait de ne pas pouvoir accepter d'entendre la langue québécoise sur scène démontre qu'une certaine catégorie de spectateurs sont encore aux prises avec les reliques du colonialisme culturel : «lls considèrent leur langue comme incapable de traduire un langage étranger de niveau équivalent." (Lefebvre at Ostiguy 1978: 47). En traduction théâtrale, le traducteur se doit de conserver le rapport texte / public et conséquemment, «il est nécessaire, si le langage populaire est employé. yue celui-ci soit celui du public. Sinon, il se produit un effet de folklorisation" (ibid.: 45).

Nous avions décidé de traduire Der Müll, die Stadt und der Tod en québécois (en Introduisant des passages en français international et en joual s'il le fallait, pour respecter les différents niveaux de langue du texte de départ et l'usage qui est fait de la langue parlée à Montréal), tout en préservant le lieu d'origine où se déroule l'action, c'est-à-dire Francfort. Cette traduction aurait préservé les traits originels du lieu de l'action. Nous uurions conservé les noms allemands et tenté de traduire les chansons le plus fidèlement possible. Voici le premier problème auquel nous avons dû être confrontée : les chansons. Une chanson fait appel a toute une gamme de connotations pour l'auditeur. En traduisant les chansons de l'allemand au français, il se produit un effet de distanciation lors de la réception puisque l'auditeur $n^{\prime}$ est pas en mesure de reconnaître quoi que ce soit : il n'y a aucun réseau de connotations possible. Il vaut mieux. à notre avis, introduire des chansons connues du public, pour que la transmission du discours thêatral ne soit pas affectée. Les chansons font partie intégrante de la performance scénique et celle-ci, comme l'affirme Pavel Campeanu (1975: 105), est «un objet à double signification». Celui-ci explique que cette double signification comprend le niveau des signes, qui sont circonscrits dans le monde du quotidien, et le niveau des symboles, «une résultante possible de l'association de ces signes" (ihid. : 105) :

Les signes sont portcurs d'information. tandis que le niveau symbolique suppose sans alternative une perception émotionnelle de nature esthétique. $|\ldots|$ Le destin du spectacle ne dépend pas de sa densité informationnelle, mais de son intensité émotionnelle $|\ldots|$ Le symbole est la transfiguration du signe. Le talent est le don de dominer les mécanismes de cette transfiguration. (ibid. : $10.5-106)$

Ce talent doit aussi, à notre avis, être partagé par le traducteur. qui doit tenter, entre autres choses, de remplacer les chansons du texte source par des chansons de la culture cible transmettant une symbolique similaire. Il y a cependant une exception à cette règle : dans 
le cas où l'auteur de la pièce aurait lui-même composé une chanson, comme c'est le cas pour la pièce de Fassbinder, qui comprend quelques lieder à la Brecht. II faut cependant remarquer qu'un metteur en scène élitiste, affectionnant particulièrement l'exotisme. pourrait éventuellement décider de conserver toutes les chansons en Allemand! On peut certes chercher à sauvegarder l'originalité nationale et culturelle d'une pièce, comme l'affirme Georges Mounin, en demandant au spectateur de faire l'effort de s'adapter. Et cela était très à la mode au Québec, dans les années quatre-vingt, mais "cette espèce de traduction restera toujours une tentative d'avant-garde, destinée à un public restreint» (Mounin 1968: 10).

Si nous optons pour des chansons connues du public montréalais, mais que les noms des personnages sont toujours allemands et que l'action se déroule toujours à Francfort, peut-on parler d'une traduction ? Où est la limite entre traduction et adaptation en traduction théâtrale ? Nous venons de démontrer qu'il faut faire appel à un réseau de connotations connu du public, notamment pour les chansons. Et, en y réfléchissant plus longuement, puisque les noms des personnages font aussi partie de ce réseau de connotations culturelles, $n{ }^{\prime}$ est-il pas incongru d'entendre des noms allemands lorsque les personnages s'expriment en québécois? II ne s'agit plus ici. à notre avis, de colonialisme culturel, contrairement à ce que pensent Lefebvre et Ostiguy (1978:44-45) : nous affirmons une fois de plus qu'il faut traduire pour le public auquel on s'adresse, donc dans les registres de la langue québécoise, mais si l'on introduit un effet d'adaptation à un certain niveau (ici, pour les chansons) pour que la communication ne soit pas entravée, ne faut-il pas poursuivre dans ce sens et transposer l'action à Montréal pour que le tout soit plus crédible pour l'auditeur, pour qu'il puisse s'y identifier et, par le fait même. comprendre davantage les intentions de lauteur" Il faut qu'il s"établisse une connivence entre l'auteur, les comédiens, le public et le traducteur. "Des publies différents appellent souvent des adaptations différentes. $|\ldots|$ Toute adaptation se fait au nom de l'authenticité, du respect de l'esprit de l'ouvre, de la préservation de sa saveur originale, mais aussi et peut-être surtout au nom du public auquel l'adaptation est destinée.» (Delisle 1986: 6). Prenons, par exemple. le cas de cette citation des frères Grimm qui est passée dans la langue allemande de tous les jours: "Ach wie gut, dass niemand weiss, dass ich Rumpelstilzchen heiss's. On ne peut certainement pas traduire ce passage puisqu'il est tout à fait incompréhensible pour quelqu'un qui n'est pas d'origine allemande ou anglaise. Il faut essayer de trouver une citation tirée d'un conte français qui rende à peu près le même sens. C'est, à notre avis. la tâche la plus ardue du traducteur littéraire et il arrive qu'on ne puisse pas trouver d'équivalent dans la langue et la culture d'arrivée. C'est pourquoi maintes traductions littéraires comprennent des coupures.

"Realistic dialogue occasionally poses insurmountable problems in translation, and sometimes I just forget about it", avouait en toute franchise le comédien. directeur artistique et traducteur Ronald Guevremont lors d'une table ronde sur l'adaptation théâtrale... (Délisle $1986: 41$

Ces coupures n'affectent souvent en rien la compréhension de l'auvre mais on ne peut plus parler de traduction. à moins d'utiliser un terme technique comme "traduction verre transparent" ou même "tradaptation», comme l'a fait le traducteur québécois Michel Garneau. Jean Delisle (ihid. : 4) considère qu' «avoir de l'oreille, être sensible au rythme des phrases, mais aussi au déroulement de l'action, manier parfaitement la langue orale sont autant de qualités qui font un bon tradaptateur». Michel Garneau n`a cependant pas transposé l'action au Québec, et c'est probablement la raison pour laquelle il a forgé ce terme. qui se veut distinct du terme péjoratif adaptation. 
À mesure qu'avance notre réflexion, force est de constater que le traducteur est obligé d'adapter en traduction théâtrale, du moins dans une certaine mesure. Cependant. pour certains traducteurs, adapter n'est pas nécessairement transposer au Québec. Qu'estce qui pourrait justifier, pour la pièce qui nous concerne, une adaptation complète de l'ouvre, c'est-à-dire sa transposition à Montréal ? Premièrement, l'intention de l'auteur : vouloir mettre à nu certaines réalités concernant la ville des spectateurs. En laissant l'action se dérouler à Francfort, le spectateur ne se sent pas concerné. L'action a lieu dans une de ces contrées lointaines au passé fasciste et sa présence au theâtre lui donne le rôle de voyeur. Si, par contre, on transpose l'action à Montréal et que l'on fait du néo-nazi allemand un skinhead québécois, le spectateur réagit et décode différemment. Il se sent concerné. Il s'identifie à une réalité qui existe chez lui. «Le grand avantage de l'adaptation est de faire appel à un réseau de connotations connu du spectateur ; ce dernier est alors en mesure de comprendre plus facilement le déroulement du spectacle et de communiquer avec les intentions de l'auteur." (Lefebvre et Ostiguy 1978:45). René-Jean Pouparı (1976: 86) est du même avis lorsqu'il écrit :

Veut-il flatter son goût pour l'exotisme au risque de ne $[\ldots]$ toucher (le public] que superficiellement, il fle metleur en scènef réclamera une version respectant la couleur locale. Entend-il, au contraire, l'atteindre en profondeur, il préférera, dans certains cas, une version "nationalisée" dont les valeurs connotatives correspondent au fond culturel de la langue d'arrivéc.

Georges Mounin (1968: 8) spécifie que l'adaptation permet de traduire «non seulement des énoncés, mais des contextes, et des situations, de façon qu'on puisse immédiatement les comprendre au point de rire ou de pleurer".

Toute langue utilisé par une ethnie est liee a une culture, au sens que les sociologues donnent a ce mot, et le respect des faits de culture s'accommode mal de la littéralité. [... Dans quelle mesure le lecteur [le spectateur] tient-il a être dépaysé, à sentir qu'il n'est pas chez lui ? Prefere-1-il au contraire que l'adaptation soit complète pour que rien ne lui rappelle l'identité aculturelle" de l'original ?" (Darbelnet 1970:93-94)

Dans bien des cas, les attentes du spectateur vont en fait dans le sens opposé. On tient souvent à vouloir se retrouver ailleurs, à sentir justement que l'action ne se déroule pas au Québec (s'il s'agit d'une pièce traduite). Mais est-il possible de traduire en québécois tout en prétendant que l'action se déroule ailleurs? En d'autres termes, la traduction "verre coloré" (avec les noms des personnages en allemand et le lieu de l'action a Francfort pour la pièce qui nous concerne) est-elle envisageable si l'on tient compte du fait que la langue parlée au Québec est la langue québécoise? Prenons l'exemple de la Iraduction américaine de Der Müll, die Stadt und der Tod par Denis Calandra. Pourquoi est-il plus facile pour le spectateur d'accepter la convention ? D'accepter que les noms des personnages soient allemands mais yu'ils parlent anglais ? Pourquoi est-il facile pour le public d'entrer dans le jeu et de croire que l'action se déroule à Francfort ? L'anglais est une langue internationale. Elle est omniprésente. Elle est parlée par plus de $\mathbf{4 . 3 4}$ millions de personnes à travers le monde. Mais qu'en est-il du québécois ? On ne peut plus parler de colonialisme culturel lorsqu'on affirme qu'il semble incongru de faire parler en québécois des personnages portant des noms allemands. La langue québécoise n'est parlée que par six millions de personnes et est spécifique au territoire exigu du Québec. Quelle autre solution s'offre a nous? Lorsque Lefebvre affirme que l'adaptation n'est peut-être qu'un truc pour justifier l'emploi du langage populaire québécois (Lefebvre et Ostiguy $1978: 32$ ), il ne s'agit pas d'un truc, mais bien d'une nécessité !

On reprochera sans doute au traducteur qui décide d'adapter une pièce de théâtre de créer «un miroir de soi plutôt qu'une fenêtre sur l'Autre» (Mailhot 1987:49). Nous 
croyons que cela dépend de la façon dont le travail est fait. Dans le cas de la pièce qui nous conceme, il est possible d'adapter sans s'éloigner du texte de départ tout en servant

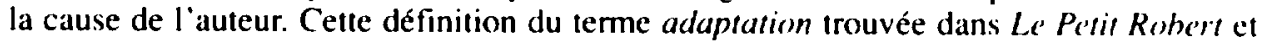
datant de 1885, mériterait d'être reformulée: "Traduction très libre d'une pièce de thêâtre, comportant des modifications nombreuses qui là mettent au goût du jour ou la rajeunissent.» (Rey 1991 : 23). Or. une adaptation - outre la deuxième acception du mol: "Transposition à la scène ou à l'écran d'une ceuvre d'un genre littéraire différent" (ibid. : 23) - ne doit justement pas être une traduction très libre comportant de nombreuses modifications. Premièrement, il serait bon de spécifier qu'adapter. c'est transposer l'action dans le pays de la culture cible et/ou transposer l'action à une autre époque : "L'adaptation peut suivre deux axes alu niveau du changement de contexte : un axe spatial et un axe temporel» (Lefebvre et Ostiguy 1978: 34). Et il est possible d’adapter tout en demeurant fidèle au texte et à la pensée de l'auteur. La définition du Petit Rohert vient justement renforcer tous nos préjugés à l'égard de l'adaptation.

Alors que Laurent Mailhot (1987: 48), à propos de ladaptation de Macheth par Garneau, se pose la question suivante: "Est-ce une nouvelle forme d'aliénation, de sujétion ?". Susan Bassnett-McGuire (1985:93) affirme qu'on ne devrait même pas faire la distinction entre adaptation et traduction et que tout ce jargon ne sert qu'à brouiller les pistes : "The distinction between a 'version' of an SL text and an 'adaptation' of that text seems to me to be a complete red herring. It is time the misleading use of these terms were set aside». À son avis, la traduction d'une pièce de théatre est tellement complexe, il faut tenir compte d'une telle multiplicité de codes, qu imposer une «bonne façon" de traduire le théâtre est un non-sens. Les Américains $n$ 'ont pas cette tendance à mépriser les adaptations théâtrales qui transposent l'action dans un coin de leur pays. favorisant ainsi l'utilisation d'un dialecte régional.

It is becoming an accepted "experiment". for example. to do Chekhov plays in a Southern American setting, on the grounds that the American language has more room for a rhetoric of self-dramatization in its warmer regions than anywhere else: once the decision about dialect is made. however, the setting, the translation of all the cultural facts and reference.s. even the substitution of emancipated slave for emancipated serf becomes almost mechanical. (Hollander 1959:228)

Nous pensons que notre adaptation de la pièce de Fassbinder "dit l'Autre" tout en «étant dite par l'Autre». (L'un n'exclut pas l'autre comme l'affirme Laurent Mailhot [1987: 48].) La "trahison" est réduite au minimum.

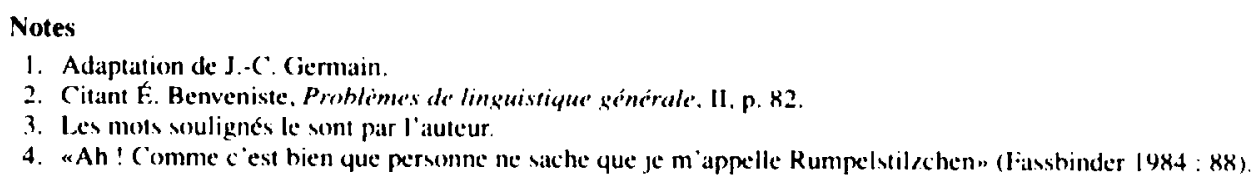

\section{REFÉRENCES}

BASSNETT-MCGUIRE, Susan (1485) : "Ways Through the labyrinth : Strategies and Methods for Translating

Theatre Texts", Hermans. T. (dir.). The Mamipularion of Laterahure. Iondres. Croom-Helm. Pp. 87.102

BÉLISI.F. Rosemarie (1986) : "Une question de niveau de langue", ( irr'un, mars. p. 9.

BÉLISL.E, Rosemarie ( 199()$)$ : "Une question de niveau de langue". ./e'u, n ' 56. pp. I 8.23

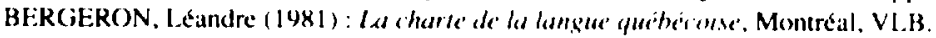

BERMAN. Antoint (1985): “La Traduction et la lette", ou "l:Auberge du kointain". Le's Fours de Babel (Exsais sur la traduction). Matuvezin. Trams-Lurop-Repress, pp. 35-125. 
BBRECHT. Benoit (1976) : Le huffer impromptu su la nôsse chez les propriefaires de hungalow', adaptation de J.C. Germain, inédit, Manuscrit de l'École nationale de theatre.

BBRISSET, Annie (1986) : "Vive la traduction... libre !», Circuir, mars, p. 10.

BRISSET, Annie (1990a) : Socioncritique de la traduction. Theatre et alterité au Quebec (1968-1988), Longueuil, Le Préambule.

BRISSET, Annie (19(4)b) : "Molière et ses traducteurs etrangers". Sixiemes assises de la traduction liffercaire (Arles 1989). Traduire le théatre. Paris. Acles Sud

BRISSET, Annie ( 1990$)$ ) : "Traduire pour s'inventer", Jeu, $n^{\circ}$ 56, pp. 55-60)

BROWER. Reuben A. (dir.) (1959) : On Translarion, Cambridge, Harward University Press.

Cahier de théatre Jeu (1990): $\mathbf{n}^{\prime \prime}$ 56. Truduction théatrale, Montreal.

CAMPEANU, Pavel (1975): „(In rôle secondaire : le spectateurw, Sémiologue de la représentation, Bruxelles, Complexe.

DARBEL.NET, J. ( 1970$)$ : “Traduction litterale ou traduction libre ?", Meta, vol. 15, n"2.

DARBELNET, J. (1976): Le francuis en contact avec l'anglais en Amérique du Nord. Quebec, Presses de I'Université Laval.

DARBELNET, J. (1977) : «Niveaux de la traduction», Babel, vol. XIII, $n^{\circ} 1$.

DELISLE. Jean (1986) : „Dans les coulisses de l'adaptation theâtrale", Circuit, $n^{\circ} 12$, mars, pp. 3-8

DEPRATS, Jean-Michel (1990) : “Traduire, adapter, écrire". Sixiemes assises de la traduction littéraire (Arle's 1989). Traduire le théâre. Paris, Actes Sud.

DURAND. Régis (1975) : „Problèmes de l'analyse structurale el sémiotique de la forme théatrale ". Sémiologie de la représensatoon. Bruxelles, Complexe.

I:CO. Umbero (1975) : «Paramètres de la sémiologie theâtrale». Sémiologie de la représentation. Bruxelles, Complexe.

FASSBINDER, Rainer Werner (1984): Die hilteren Tränen der Petra von Kant / Der Müll. die Stadt und der Tod. Frankfurt am Main, Verlag der Autoren.

FASSBINDER, Rainer Werner (198.5): Garbage. The City and Death, Iraduction de Denis Calandra. New York, PAJ.

GAGNON, Lysiane (1989) : "Our, mais quelle langue ?". La Presse. 1" avril, p. B3.

GINGRAS. René (1990) : «Le statut du québecois comme langue de traduction», Jeu, no 56, pp. 25-37.

HELBO. André (dir.) (1975) : Sémologie de la représcntatuon, Bruxelles. Complexe.

HELBO. André (1983) : Les mots et les gestes. Essui sur le théâtre, Lille. Presses Universitaires de Lille.

1 HOLL.ANDER, John (1959) : "Versions, Interpretations, Performancesw, On Translation. Cambridge. Harward University Press.

IAKOBSON, Roman, (1959) : “On Linguistic Aspects of Translation ". On Translation, Cambridge, Harvard University Press.

JOURDHEUIl. Jean (1990) : "Traduire. adapter. ccrire". Sixiemes assises de la traduction lifteraire (Arles 1989). Traduire le thédire. Paris. Actes Sud.

LALONDE, Michele (1979): Défenser et illustration de la langue québécorise. Paris, Seghers-Laffont, coll. “Change".

LEBRUN-SLDRY (1947) : Shakespeare, Le Marchand de Venise, Iraduction de Mme Lebrun-Sudry, Paris, Rivade.

LEFEB VRE. Paul et Pierte OSTIGUY (1978) : “L'adaptation theâtrale au Québec ${ }^{\prime}, J e u, n^{\circ}$ 9, pp. 32-47.

L.FFEVERE. André (1981): "Beyond the Process: Literary Translation in Literature and Literary Theory», Trunslation Spectrum. Essavs in Theory and Practice. Albany, State University of New York Press. pp. $52-59$.

LEON, Monique (1991) : Evercuess systrmatiques de prononciation framsaise, Paris, Hachette.

L.ÉVESQUE. Roten ( 1993 ) : "Entre chien el loup», Le Devoir. 2 juin. p. AX.

MAlL.HOT, Laurent (1987): "Traduction et "Nontraduction" : l'épreuve du voisin etranger dans la litterature québécoise ". l' altérité dans la litlérature quéhéconse', Bologne, CL.UEB, pp. 13-59.

MARCUS, Salomon (197.5) : "Stratégies des personnages dramatiques". Sémiologie de la représention. Bruxelles, Complexe

MOLIĖRE, J.-B. (1977) : Le's Fuax Brillants de Félix-Gahriel Marchand, adaptation du Bourgeonis gentilhomme par Jean-Claude (iermain. Montréal, VLB éditeur.

MOUNIN, (ieorgen (1455): Le's helle's infideles. Paris, Cahiers du Sud.

MOUNIN, (ieorges $(1968)$ : “l a traduction au theâtrem. Babel, vol. XIV, n' 1, pp. 7-11.

PAVIS. Patrice (1987) : Dictionnuire du thédrre. Paris, Messidor / Éditions Sociales.

PAVLOVIC, Diane (1987) : "Cartographie : I'Allemagne québecoise". Jeu. ${ }^{\circ} 43$. pp. 77-110 
POGGIOLI. Renato (1959): «The Added Artificer». On Translation, Cambridge, Harvard University Prews.

POULIN MIGNAUIT, Hélène et (jilles $S I X(199(0)$ : Le françuis un Québec'. Montréal, Sodilis.

POLIPART, Rene-Jean (1976): "Communication theâtrale et traduction", Cahiers internatumaux de wmbolisme, $\mathrm{n}^{\circ} 31-32, \mathrm{pp} .77-8 \mathrm{x}$.

REY, A. et J. REY-DEBOVE ( 1991$)$ : Le Pe'ut Rohert, Paris, Dictionnaires Le Roben.

SHAKESPEARE (1947): Le Marchand de Vensse, traduction de Mme Lebrun-Sudry, Paris, Rivade.

SHAKESPEARE: (1978): Machecth, traduction de Michel Garncau, Montréal. VLB.

Siviemes assises de la sraduction lintiraire (Arles 1989). Truddure le théatre (1990) : Paris. Actes Sud.

THIEBER(il:R. Richard (1972) : “Le langage de la traduction», Langages, n” 28. p. 77.

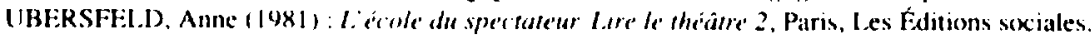

WFILLWARTH, George E. (1981): “Special Considerations in Drama Translation». Translatum Spectrum.

Eswass in The'ory and Practice, Alhany. State University of New York Press, pp. 140-146. 